\title{
On the extremality bound of stringy black holes
}

\author{
Pablo A. Cano, ${ }^{a}$ Tomás Ortín $^{a}$ and Pedro F. Ramírez ${ }^{b}$ \\ ${ }^{a}$ Instituto de Física Teórica UAM/CSIC, \\ C/ Nicolás Cabrera, 13-15, C.U. Cantoblanco, 28049 Madrid, Spain \\ ${ }^{b}$ INFN - Sezione di Milano, \\ Via Celoria 16, I-20133 Milano, Italy \\ E-mail: pablo.cano@uam.es, tomas.ortin@csic.es, \\ ramirez.pedro@mi.infn.it
}

ABSTRACT: A mild version of the weak gravity conjecture (WGC) states that extremal black holes have charge-to-mass ratio larger or equal than one when higher-curvature interactions are taken into account. Since these corrections become more relevant in the low-mass regime, this would allow the decay of extremal black holes in terms of energy and charge conservation. Evidence in this direction has been mainly given in the context of corrections to Einstein-Maxwell theory. Here we compute corrections to the charge-to-mass ratio of some dyonic extremal black holes explicitly embedded in the heterotic string effective theory. We find that modifications of the extremality bound depend on the solution considered, with the charge-to-mass ratio remaining unchanged or deviating positively from one. Additionally, we observe that the introduction of the higher-curvature terms increases the Wald entropy in all cases considered, whose variation does not seem to be correlated with the charge-to-mass ratio, contrary to the situation in Einstein-Maxwell theory.

Keywords: Black Holes in String Theory, Superstrings and Heterotic Strings

ARXIV EPRINT: 1909.08530 


\section{Contents}

1 Introduction 1

2 A family of extremal black holes 4

2.1 Supergravity zeroth-order solution 5

2.2 Case 1: $q_{V}<0 \quad 6$

2.3 Case $2: q_{V}>0 \quad 8$

$\begin{array}{llr}3 & \text { Discussion } & 9\end{array}$

$\begin{array}{ll}\text { A The heterotic theory } & 11\end{array}$

B Solving the equations of motion $\quad 12$

$\begin{array}{ll}\text { C Wald entropy } & 14\end{array}$

$\begin{array}{ll}\text { D Case 3: } q_{A} \cdot q_{V}=0 & 16\end{array}$

\section{Introduction}

Black holes have played, and continue to play, a central role in fundamental aspects of physics. Cutting-edge advances in the understanding of quantum gravitational aspects of string theory have been possible thanks to their study [1-9]. In recent years some attention has been dedicated to the problem of whether extremal black holes, those with vanishing temperature, should decay or remain as stable states. The question arises because, in many situations, black hole remnants are regarded as problematic. For example, it can be argued that a theory of gravity with global symmetries contains an infinite number of remnants below a certain mass scale, which is usually considered to be inconsistent $[10,11]$. On the other hand, stable black holes of theories with local symmetries are not infinitely degenerate (under a certain mass scale), so it is more difficult to produce arguments against their existence. Still, they are associated to an infinite tower of charged states not protected by a symmetry principle, and it was conjectured in [12] that a finiteness criterion should be applied such that those are unstable.

Hence, we are led to consider the decay of extremal black holes. The simplest of those is the Reissner-Nordström one, which has $M=Q$ in appropriate units. It is immediate to see that decay of this black hole in two separated states with $q_{1}+q_{2}=Q, m_{1}+m_{2} \leq M$ requires $q_{i} / m_{i} \geq 1$ for at least one of them. ${ }^{1}$ These bounds can be saturated in the special

\footnotetext{
${ }^{1}$ This is a necessary but not sufficient condition for the process to occur, which seems thermodynamically disfavored. Since these black holes have zero temperature, standard Hawking radiation does not take place.
} 
case in which there is no binding energy between the products, while a strict inequality is expected in generic situations.

The weak gravity conjecture proposes that the spectrum of a quantum theory of gravity must be such that extremal black holes can decay, as far as energy and charge conservation are concerned. There are two possible scenarios compatible with the conjecture. Under the rather reasonable assumption that the decay would occur through the emission of a light particle by the black hole, i.e. $m_{1} \gg m_{2}$, the consequences of having $q_{2} / m_{2}>1$ are stronger than those of the complementary scenario $q_{1} / m_{1}>1$ in the following sense. In terms of the former, the WCG becomes a sharp tool that can be useful to discern if an effective theory belongs or not to the swampland $[12,13]$. Examples of applications of the conjecture in this direction can be found in [14] and references therein. On the other hand, the latter possibility corresponds to a milder version of the conjecture that would just provide information about states far heavier than the Planck mass, and hence it is less useful for the swampland program. Of course, the two scenarios are not mutually exclusive, and it is conceivable that they might be related [15].

The problem of computing corrections to the extremal charge-to-mass bound has been considered before by other authors in several frameworks. To date, the Reissner-Nordström black hole of Einstein-Maxwell theory supplemented by higher-derivative terms is arguably the system which is best understood in that respect. An explicit computation for that system that gives the corrections to the ratio in terms of the value of the coefficients of the higher-derivative terms was presented in [16] (see also [17]). Subsequent works have proposed that, demanding analyticity of scattering amplitudes, unitarity and causality constrain these coefficients such that there is a positive deviation of the charge-to-mass ratio of extremal black holes [18-20]. The same conclusion has been reached from the analysis of the renormalization of one-loop divergences [21]. Likewise, it has been proposed that this can be related to the positivity of the corrections to the entropy of the black hole induced by higher-derivative operators [22-24]. The study of black holes in Einstein-Maxwell theory is well justified and interesting, as it provides a relatively simple arena to explore this question while making contact with dominant interactions in real world experiences. On the other hand, since the problem at hands is intimately related to quantum gravity, it is important to ask if the positive character of the deviation is displayed by models with an explicit string theory embedding.

Effective gravitational theories derived from string theory usually contain, besides vectors, scalars. Einstein-Maxwell-Dilaton (EMD) theory arises as a natural truncation of the effective theories of different string models. For instance, it appeared as a truncation of $N=4, d=4$ supergravity $^{2}$ in [25], where the first EMD black-hole solutions where found. These solutions were later rederived and studied in [26, 27]. ${ }^{3}$ The Kaluza-Klein theory obtained by compactifying the 5-dimensional Einstein-Hilbert action on a circle also provides another particular example of EMD theory with the Kaluza-Klein scalar playing the role of dilaton field. Different instances of EMD theory are distinguished by the different couplings of the dilaton to the vector field kinetic term in the action.

\footnotetext{
${ }^{2}$ This is the effective theory of the Heterotic Superstring compactified on $T^{6}$.

${ }^{3}$ This solution is usually referred to as the GHS black hole.
} 
Although EMD theory looks very similar to Einstein-Maxwell (EM), there are important differences. In particular, the purely electrically or magnetically charged ReissnerNordström black hole with constant scalar is not a solution of the equations of motion, as the vector field acts as a source for the dilaton. Only when the black hole is dyonic with equal magnetic and electric charges the source term in the dilaton equation vanishes and the dilaton can take a constant value. For generic values of the electric and magnetic charges, one gets charged black holes with a non-trivial scalar field.

In the extremal limit, these black holes are regular, except in the purely electric and purely magnetic cases. Corrections to the charge-to-mass ratio in one of these singular cases have been studied in [16], although, due to the singularities, this is not a good ground to discuss stability of extremal black holes. Still, it is worth mentioning that the correction has again a positive character, which gives some support to the mild WGC.

However, the positive deviation of the charge-to-mass ratio cannot be general in string theory. Supersymmetric black holes are special. When they are regular they necessarily carry several charges and their mass is given by a linear combination of them with modulidependent coefficients. Typically, the scalars are not constant, but their value at the horizon is fixed in terms of the charges due to the attractor mechanism [2, 5, 6, 28-30]. The linear relation between mass and charge is a salient feature of supersymmetric systems and, as supersymmetric black holes are extremal [1], the charge-to-mass ratio can be expected to remain unmodified by higher-derivative corrections. This has been recently shown to be the case in three- and four-charge heterotic black holes $[31,32]$ in, respectively, five and four dimensions. ${ }^{4}$ Nevertheless, one should notice that these configurations correspond to a bound state of a (large) number of fundamental objects without binding energy. This means that, speaking in terms of energy and charge conservation, the decay of supersymmetric black holes is possible. Another family of configurations of $\mathcal{N} \geq 2$ four-dimensional supergravity for which no corrections to the ratio have been observed (even at one-loop quantum level and non-supersymmetric solutions) was recently described [21]. These black holes are obtained through a particular embedding of dyonic solutions of Einstein-Maxwell theory for which the solution is claimed to not receive corrections at all [33].

In this article we compute explicitly the first-order $\alpha^{\prime}$ (fourth order in derivatives) corrections to the charge-to-mass ratio of the extremal Reissner-Nordström black hole embedded in heterotic string theory in several ways. All the embeddings that we will consider here are dyonic (so the dilaton vanishes at lowest order in $\alpha^{\prime}$ ) and non-supersymmetric (so there is a chance of having non-vanishing $\alpha^{\prime}$ corrections). To the best of our knowledge, these provide the first examples in which such a computation has been made using a explicit embedding of the black-hole solutions in a superstring theory whose first-order in $\alpha^{\prime}$ corrections are explicitly known in detail.

We start in section 2 with a description of the zeroth-order solutions we start from. They are 2-vector dyonic, extremal Reissner-Nordström black holes, although we take only one of the charges to be independent for simplicity. Depending on the election of the

\footnotetext{
${ }^{4}$ In those articles, it was noted that the relation between mass and the number of fundamental constituents of the black hole is modified by $\alpha^{\prime}$-corrections. However, the relation between mass and asymptotic charges remains unchanged.
} 
relative signs of the charges, there are two families of solutions that can be considered, for which the consequences of including the higher-curvature corrections are different. In one case the charge-to-mass ratio of the solution remains unchanged, while in the other it deviates positively from one. On the other hand, the Wald entropy of both solutions is equal and differs from the expression of the zeroth-order system. Our results are discussed in section 3 .

In the different appendices we include the details about the different computations that we have performed. The effective field theory of the heterotic string at first order in $\alpha^{\prime}$ is described in appendix A. The equations of motion evaluated for the spherically-symmetric ansatz can be found in appendix B and the calculation of the Wald entropy is addressed in appendix C. In appendix D we compute the corrections for a solution different to the ones considered in section 2 , which has some peculiar features.

\section{A family of extremal black holes}

Let us consider the following field configuration of heterotic superstring theory, whose perturbative action and equations of motion are briefly reviewed in appendix A,

$$
\begin{aligned}
d \hat{s} & =e^{2\left(\phi-\phi_{\infty}\right)} d s^{2}-c^{2}\left(d z+V / c_{\infty}\right)^{2}-d y^{i} d y^{i}, \\
\hat{H} & =F \wedge\left(c_{\infty} d z+V\right)+H, \\
e^{-2 \hat{\phi}} & =\frac{1}{c} e^{-2 \phi},
\end{aligned}
$$

where $d s^{2}$ is the four-dimensional metric in the Einstein frame, $F$ is a 2 -form, $V$ is a Kaluza-Klein vector, $c$ is a KK scalar, $H$ is a 3 -form, and $\phi$ is the four-dimensional dilaton. In addition, $c_{\infty}$ and $\phi_{\infty}$ are the asymptotic values of $c$ and $\phi$. These are effective fourdimensional fields, while hatted objects represent ten-dimensional fields of the heterotic theory. This ansatz corresponds to the compactification of heterotic superstring theory on $\mathbb{S}_{z}^{1} \times \mathbb{T}^{5}$, where we truncate all the fields that have indices on $\mathbb{T}^{5}$, while the KK reduction on $\mathbb{S}_{z}^{1}$ is general. The coordinates parametrizing the compact space $z$ and $y^{i}, i=1,2,3,4,5$ have all period $2 \pi \ell_{s}$.

At the supergravity level (zeroth-order in $\alpha^{\prime}$ ), this gives rise to the following fourdimensional effective theory

$$
\begin{aligned}
S=\frac{1}{16 \pi G_{N}^{(4)}} \int d^{4} x \sqrt{|g|}\left\{R+2(\partial \phi)^{2}+\right. & \frac{(\partial c)^{2}}{c^{2}}+\frac{e^{-4\left(\phi-\phi_{\infty}\right)}}{2 \cdot 3 !} H^{2} \\
& \left.+\frac{e^{-2\left(\phi-\phi_{\infty}\right)}}{4}\left(G^{2}+\frac{c_{\infty}^{2}}{c^{2}} F^{2}\right)\right\}
\end{aligned}
$$

where $G=d V$ and, at this order, $F=d A$. The four-dimensional Newton's constant is given by

$$
G_{N}^{(4)}=\frac{G_{N}^{(10)}}{c_{\infty}\left(2 \pi \ell_{s}\right)^{6}}
$$


Also, the 3-form satisfies the Bianchi identity

$$
d H=-F \wedge G,
$$

and using it we can dualize $H$ into a scalar field. From the effective four-dimensional action (2.2) one sees that, at the supergravity level, one could truncate $V, H$ and $c$. This would simplify the system to the Einstein-Maxwell-Dilaton model. However, it turns out that this is inconsistent once $\alpha^{\prime}$ corrections are taken into account, as those introduce nontrivial couplings between these fields. In other words, higher-derivative corrections to the Einstein-Maxwell-Dilaton effective model in the context of string theory may require the activation of additional fields. This is a well-known but often forgotten fact [34, 35].

\subsection{Supergravity zeroth-order solution}

A generalized version of the extremal Reissner-Nordstrom black hole can be a solution of the theory (2.2) if we allow for dyonic vectors. Let us consider the following configuration,

$$
\begin{aligned}
d s^{2} & =\left(1+\frac{Q}{r}\right)^{-2} d t^{2}-\left(1+\frac{Q}{r}\right)^{2}\left(d r^{2}+r^{2} d \Omega_{(2)}^{2}\right) \\
A & =\frac{2 q_{A}}{(r+Q)} d t-2 p_{A} \cos \theta d \varphi \\
V & =\frac{2 q_{V}}{(r+Q)} d t-2 p_{V} \cos \theta d \varphi \\
\phi & =\phi_{\infty}, \quad c=c_{\infty}, \quad H=0
\end{aligned}
$$

where $q_{A, V}, p_{A, V}$ are the electric and magnetic charges of the vectors $A$ and $V$ (in Planck units) and $Q=\sqrt{q_{A}^{2}+p_{A}^{2}+q_{V}^{2}+p_{V}^{2}}$ is the total duality-invariant charge. ${ }^{5}$ On the other hand, the mass of this black hole is $M=Q$. It is easy to check that this is a solution of (2.2) if the charges satisfy the following conditions

$$
\begin{gathered}
\left|q_{A}\right|=\left|p_{A}\right|, \quad\left|q_{V}\right|=\left|p_{V}\right|, \\
q_{A} p_{V}+p_{A} q_{V}=0 .
\end{gathered}
$$

The first two conditions ensure that $F^{2}=G^{2}=0$ while the third one implies that $F \wedge G=0$, and in this way the scalar fields have no sources. This special point in charge space has the property that the scalars are trivial at the supergravity level, although this does not hold once higher-curvature corrections are implemented, as we show below. Let us note that starting from a given solution, the transformation $t \rightarrow-t$ generates a new solution with opposite values of the electric charges and in turn $\phi \rightarrow-\phi$ changes the sign of the magnetic charges. Thus, without loss of generality we can consider $q_{A}=p_{A}>0$, and in that case $p_{V}=-q_{V}$. Hence, there are two inequivalent sets of solutions, corresponding to $q_{V}>0$ and $q_{V}<0$. We wish to compute the first-order $\alpha^{\prime}$-corrections to these solutions, but for simplicity we will restrict to the case in which the absolute value of all the charges

\footnotetext{
${ }^{5}$ Invariant under electric-magnetic duality and T-duality.
} 
is the same. Hence, there are two possibilities: $q_{A}=q_{V}=p_{A}=-p_{V}=Q / 2$ and $q_{A}=-q_{V}=p_{A}=p_{V}=Q / 2$. In addition, the special case with $q_{V}=0$, in which $\alpha^{\prime}$ corrections seem to introduce pathologies in the extremal limit, is treated in appendix D.

Thus, in this article we study the corrections to a stringy Reissner-Nordström black hole which, despite having two independent $\mathrm{U}(1)$ dyonic vector fields, has only one independent charge $Q$. The configuration (2.5) has an event horizon at $r=0$, with near-horizon geometry $A d S_{2} \times S^{2}$ and it is therefore a black hole with vanishing temperature. On the other hand, the configuration does not preserve any supersymmetry, this being related to the presence of dyonic vectors, as in ref. [36].

\subsection{Case 1: $q_{V}<0$}

Let us first consider the case $q_{A}=-q_{V}=p_{A}=p_{V}=Q / 2$. Starting from the zeroth-order solution (2.5) and using (2.1), it is possible to compute the first higher-curvature corrections by solving perturbatively the ten-dimensional equations of motion at first order in $\alpha^{\prime}$, which can be found in appendix A. The details about the resolution of those equations are shown in appendix B. We find the following solution

$$
\begin{aligned}
d s^{2} & =\left(1+\frac{Q}{r}+\frac{\alpha^{\prime} Q^{2}}{8(r+Q)^{3} r}\right)^{-2} d t^{2}-\left(1+\frac{Q}{r}+\frac{\alpha^{\prime} Q^{2}}{8(r+Q)^{3} r}\right)^{2}\left(d r^{2}+r^{2} d \Omega_{(2)}^{2}\right) \\
F & =\frac{Q}{(r+Q)^{2}}\left(1+\frac{\alpha^{\prime} Q^{2}}{4(r+Q)^{4}}\right) d t \wedge d r+Q\left(1+\frac{\alpha^{\prime} Q(Q+4 r)}{2(r+Q)^{4}}\right) \sin \theta d \theta \wedge d \varphi \\
V & =-\frac{Q}{(r+Q)} d t-Q \cos \theta d \varphi \\
\hat{\phi} & =\hat{\phi}_{\infty}+\frac{\alpha^{\prime} Q^{2}}{4(r+Q)^{4}}, \\
c & =c_{\infty}\left(1+\frac{\alpha^{\prime} Q^{2}}{4(r+Q)^{4}}\right), \quad H=0 .
\end{aligned}
$$

The conditions that we have imposed in order to solve the equations of motion are the same than those imposed for the original supergravity solution, namely

- regularity of the event horizon located at $r=0$,

- fixed asymptotic value of the scalars: $\hat{\phi} \rightarrow \hat{\phi}_{\infty}$ and $c \rightarrow c_{\infty}$,

- the metric is asymptotically flat,

- absence of additional free charges at order $\alpha^{\prime}$.

The last point means that we do not introduce artificial shifts in the charges. In fact, performing a transformation of the form $Q \rightarrow Q+\alpha^{\prime} \delta Q$ in the original solution generates a new solution which, apparently, contains $\alpha^{\prime}$-corrections. The integration constants of the equations of motion have to be appropriately chosen so that this type of shift does not occur.

Observe that in the previous solution, $V$ contains no corrections. Also, note that $F$ is not a closed form, $d F \neq 0$, so its local expression is no longer given by the exterior 
derivative of the vector field $A$. This is due to the form of the decomposition (2.1) and to the fact that $H$ is not a closed 3 -form at first order in $\alpha^{\prime}$. Thus, $F$ will have an expression of the form $F=d A+\alpha^{\prime} W$ for some 2-form $W$. Nevertheless, the correct identification of the charges carried by these vector fields can be expressed in terms of $F$ and $G$ as follows, ${ }^{6,7}$

$$
\begin{aligned}
q_{A} & =\frac{1}{8 \pi} \int_{\mathbb{S}_{\infty}^{2}} \frac{c_{\infty}^{2}}{c^{2}} e^{-2\left(\phi-\phi_{\infty}\right)} \star F, \\
p_{A} & =\frac{1}{8 \pi} \int_{\mathbb{S}_{\infty}^{2}} F, \\
q_{V} & =\frac{1}{8 \pi} \int_{\mathbb{S}_{\infty}^{2}} e^{-2\left(\phi-\phi_{\infty}\right)} \star G, \\
p_{V} & =\frac{1}{8 \pi} \int_{\mathbb{S}_{\infty}^{2}} G .
\end{aligned}
$$

We have checked that the evaluation of the integrals yields $q_{A}=p_{A}=-q_{V}=p_{V}=Q / 2$, so that the charges of the solution are indeed unmodified and $Q$ is the total charge. One might think that corrections to the charges should not be expected, as they are defined by asymptotic integrals, where the curvature goes to zero. However, there are many examples of solutions for which higher-curvature interactions behave as delocalized sources of charge [31, 32, 37, 38], and hence it is always convenient to perform this computation.

The ADM mass of the black hole can be read from the asymptotic expansion of the metric according to

$$
\lim _{r \rightarrow \infty} g_{r r}=1+\frac{2 M}{r}+\ldots
$$

From (2.7) on sees that $M=Q$, so the charge-to-mass ratio of this extremal black hole is not modified at this order,

$$
\frac{Q}{M}=1+\mathcal{O}\left(\alpha^{\prime 2}\right)
$$

It is also interesting to compute the correction to the entropy of this black hole. The application of Wald's formula for this family of solutions of the heterotic theory is described in appendix C. Upon evaluation of the resulting expression (C.10) for the background (2.7) we get

$$
\mathbb{S}=\frac{\pi}{G_{N}^{(4)}}\left(Q^{2}+\frac{\alpha^{\prime}}{4}\right) .
$$

The first term in the expression coincides with the value of the entropy before the corrections are incorporated, for a fixed value of the charge $Q$. Therefore, we find a positive

\footnotetext{
${ }^{6}$ These integral expressions for the charges are valid in the asymptotic sphere $\mathbb{S}_{\infty}^{2}$. At a generic sphere some of the expressions contain additional higher-derivative terms, which vanish asymptotically.

${ }^{7}$ In the case of the charges associated with $F$, these can be written in terms of the ten-dimensional Kalb-Ramond field strength as follows,

$$
q_{A}=\frac{g_{s}^{2}}{8 \pi\left(2 \pi \ell_{s}\right)^{5}} \int_{\mathbb{S}_{\infty}^{2} \times \mathbb{T}^{5}} e^{-2 \hat{\phi}} \star \hat{H},
$$$$
p_{A}=\frac{1}{8 \pi c_{\infty}\left(2 \pi \ell_{s}\right)} \int_{\mathbb{S}_{\infty}^{2} \times \mathbb{S}^{1}} \hat{H} .
$$ 
contribution to the entropy, that can be interpreted as capturing additional microscopic degrees of freedom that are frozen in the truncation to the two-derivative supergravity theory. At the computational level, this deviation is originated from an increase in the area of the event horizon, while the contributions in Wald's formula coming explicitly from the higher-derivative terms vanish.

\subsection{Case 2: $q_{V}>0$}

Let us now consider the case with $q_{A}=q_{V}=p_{A}=-p_{V}=Q / 2$. The first-order in $\alpha^{\prime}$ corrections turn out to be quite different. The solution reads

$$
\begin{aligned}
d s^{2} & =A^{2}\left(1+\frac{Q}{r}\right)^{-2} d t^{2}-B^{2}\left(1+\frac{Q}{r}\right)^{2}\left(d r^{2}+r^{2} d \Omega_{(2)}^{2}\right) \\
F & =\frac{Q}{(r+Q)^{2}}\left(1+\alpha^{\prime} \frac{3 Q^{2}-10 Q r-3 r^{2}}{120(r+Q)^{4}}\right) d t \wedge d r+Q\left(1-\frac{\alpha^{\prime} Q^{2}}{2(r+Q)^{4}}\right) \sin \theta d \theta \wedge d \varphi \\
V & =\frac{Q}{(r+Q)}\left(1-\alpha^{\prime} \frac{r(63 Q+r)}{120(r+Q)^{4}}\right) d t+Q \cos \theta d \varphi, \\
\hat{\phi} & =\hat{\phi}_{\infty}-\frac{\alpha^{\prime} r\left(19 Q^{2}+12 Q r+3 r^{2}\right)}{60 Q(r+Q)^{4}} \\
c & =c_{\infty}\left(1-\frac{\alpha^{\prime} Q^{2}}{4(r+Q)^{4}}\right), \quad H=0,
\end{aligned}
$$

where

$$
\begin{aligned}
& A=1+\alpha^{\prime} \frac{6 Q^{3}+13 Q^{2} r+8 Q r^{2}+2 r^{3}}{40 Q(r+Q)^{4}}, \\
& B=1-\alpha^{\prime} \frac{5 Q^{3}+9 Q^{2} r+7 Q r^{2}+2 r^{3}}{40 Q(r+Q)^{4}} .
\end{aligned}
$$

In order to obtain this solution we have imposed the same conditions as in the previous case. Notice that now $V$ receives corrections, while $F$ is again not closed. Still, one can check that the charges have the correct values $q_{A}=p_{A}=q_{V}=-p_{V}=Q / 2$, and therefore, $Q$ is indeed the total charge. We also note that, unlike in the previous solution, the dilaton acquires a non-trivial charge that cannot be removed, and it reads ${ }^{8}$

$$
Q_{\hat{\phi}}=\frac{\alpha^{\prime}}{20 Q}
$$

On the other hand, the metric component $g_{r r}$ behaves asymptotically as

$$
g_{r r}=1+\frac{1}{r}\left(2 Q-\frac{\alpha^{\prime}}{10 Q}\right)+\ldots
$$

and therefore, the mass receives corrections in this case

$$
M=Q-\frac{\alpha^{\prime}}{20 Q}+\mathcal{O}\left(\alpha^{\prime 2}\right) .
$$

\footnotetext{
${ }^{8}$ It is identified by the asymptotic expansion $\hat{\phi}=\hat{\phi}_{\infty}-Q_{\hat{\phi}} / r+\mathcal{O}\left(1 / r^{2}\right)$.
} 
Correspondingly, the extremal charge-to-mass ratio is modified

$$
\frac{Q}{M}=1+\frac{\alpha^{\prime}}{20 M^{2}}+\mathcal{O}\left(\alpha^{\prime 2}\right)
$$

and it is larger than one, in agreement with the mild form of the WGC. It is important to notice that the deviation from one becomes larger as the mass of the black hole decreases, which would allow the decay of the black hole (in terms of energy and charge conservation) without the need to emit a superextremal particle.

The Wald entropy of this solution is obtained from the evaluation of (C.10). In this case there is a negative contribution from the modification of the area of the event horizon as well as a positive one from the higher-derivative terms. The result reads

$$
\mathbb{S}=\frac{\pi}{G_{N}^{(4)}}\left(Q^{2}+\frac{\alpha^{\prime}}{4}\right),
$$

which surprisingly enough, coincides with the value found in the previous case, even though the rest of the properties of the solution are different.

\section{Discussion}

In this article we have analyzed the effect produced by higher-curvature corrections to a family of extremal, non-supersymmetric black holes in the context of heterotic superstring theory. We have found the first example of modification of the charge-to-mass ratio of an extremal black hole explicitly embedded in string theory. This example defies previous expectations that the charge-to-mass ratio of extremal black holes in a supersymmetric theory is not modified by higher-curvature corrections. Likewise, we have presented evidence that such modifications are not necessarily in correspondence with the corrections to the entropy of the black hole, as argued in refs. [22-24]. While this differs from the result of the explorations performed in Einstein-Maxwell theory, it agrees with earlier results on the supersymmetric three- and four-charge systems of the heterotic theory, as we mentioned in the introduction. The difference with the results in refs. [22, 23] can be understood if one takes into account two facts. First of all, topological terms such as the Gauss-Bonnet invariant - which implicitly appears in the heterotic string effective action - modify the black hole entropy while keeping the solution unchanged. Thus, in this case corrections to the entropy are independent from deviations of the extremal charge-to-mass ratio. On the other hand, the models considered in the previous literature do not include scalars, which are a key ingredient of stringy effective actions. As we have seen in the examples presented, these scalars are activated by higher-derivative corrections even if they are trivial in the zeroth-order solution. Scalar fields usually affect the thermodynamic description of black holes — see e.g. [39] — and it would be interesting to explore whether this could modify the conclusions of $[22,23] .^{9}$

\footnotetext{
${ }^{9}$ The fact that corrections to the entropy and the charge-to-mass ratio are not necessarily correlated has been clarified in [35].
} 
The string coupling constant and the curvature can be kept sufficiently small in the exterior region of the black hole for the cases we have considered, hence the low energy field theory description gives a good approximate description of the system. In our analysis, we focused on the case $\left|q_{A}\right|=\left|q_{V}\right|$ for simplicity, but it would be interesting to study the corrections to the solution (2.5) for general values of $q_{A}$ and $q_{V}$, so that the two dyonic vectors are independent. In that case, we expect that the extremality bound will be modified according to

$$
\frac{Q}{M} \leq 1+\frac{\alpha^{\prime}}{M^{2}} f\left(q_{A}, q_{V}\right)+\mathcal{O}\left(\alpha^{\prime 2}\right),
$$

where $f$ is a certain homogeneous function of degree 0 of the charges $q_{A}$ and $q_{V}$, which, according to the WGC, should be non-negative. In this paper, we have shown that

$$
f(q,-q)=0, \quad f(q, q)=\frac{1}{20} .
$$

In addition, in appendix $\mathrm{D}$ we consider the case $q_{V}=0$ and we show that $f(q, 0)=$ $\frac{1}{80} \cdot{ }^{10}$ Furthermore, since the result must be invariant under T-duality we conclude that $f(0, q)=f(q, 0)$. Given the values found, it is an interesting problem to search for the general expression of the function $f\left(q_{A}, q_{V}\right)$ and to check its non-negativity.

An important piece in our analysis is that the higher-curvature corrections to the theory are directly taken from the ten-dimensional heterotic string theory. This differs from the approach usually taken in the literature, where given a four-dimensional effective theory, all possible four-derivative terms that can be constructed with the corresponding field content are considered. As we have mentioned in the main text, consistency may require to enlarge the field content of an effective theory when perturbative corrections are being considered. Of course, the details depend on the UV theory on which the system is embedded. It is interesting to mention here the example of the dyonic Reissner-Nordstrom black hole solution of Einstein-Maxwell-Dilaton theory embedded in the heterotic theory $(f(q, 0)$ configuration), which requires the activation of additional fields that can be truncated at the two-derivative level [35].

The mild version of the WGC affects solutions well above Planck mass. Hence, it has little predictive power about low energy effective theories in itself. It has been recently suggested that it might be possible to relate the mild and strong versions of the WGC using modular invariance of string theory [15]. Given the growing amount of evidence in favor of the mild WGC, this seems a promising idea and it would be interesting to test it for a regular extremal black hole system of string theory. ${ }^{11}$

\section{Acknowledgments}

This work has been supported in part by the INFN, the MCIU, AEI, FEDER (UE) grant PGC2018-095205-B-I00 and by the Spanish Research Agency (Agencia Estatal de Investi-

\footnotetext{
${ }^{10}$ The study of the non-extremal black holes of that type will be the object of a coming publication [35]. $\alpha^{\prime}$-corrections seem to introduce pathologies in the extremal case even though the zeroth-order solution is regular.

${ }^{11}$ Reference [15] considered two-charge black holes, which have singular horizon in the extremal limit even after higher-curvature corrections are included [37].
} 
gación) through the grant IFT Centro de Excelencia Severo Ochoa SEV-2016-0597. The work of PAC is funded by Fundación la Caixa through a "la Caixa - Severo Ochoa" International pre-doctoral grant. PFR would like thank the Albert Einstein Institute at Potsdam for hospitality while this work was being completed. TO wishes to thank M.M. Fernández for her permanent support.

\section{A The heterotic theory}

In this article we work in the context of the heterotic superstring effective action at first order in the $\alpha^{\prime}$ expansion as constructed in [40]. We consider black hole solutions with small string coupling $g_{s}$ and sufficiently large horizon such that the supergravity approximation is valid at least outside the event horizon. Still, we are interested in looking at the information that the dominant corrections of higher order in curvature produce in some properties of the solutions. The effective action of the heterotic superstring at first order in $\alpha^{\prime}$ is given by

$$
S=\frac{g_{s}^{2}}{16 \pi G_{N}^{(10)}} \int d^{10} x \sqrt{|\hat{g}|} e^{-2 \hat{\phi}}\left\{\hat{R}-4(\partial \hat{\phi})^{2}+\frac{1}{2 \cdot 3 !} \hat{H}^{2}-\frac{\alpha^{\prime}}{8} \hat{R}_{\left.(-) \mu \nu{ }_{b} \hat{R}_{(-)}{ }^{\mu \nu b}{ }_{a}+\ldots\right\}} .\right.
$$

We use hats to denote the ten-dimensional heterotic fields. We have not included YangMills fields in the theory for simplicity. ${ }^{12}$ The curvature of the torsionful spin connection, defined as $\hat{\omega}_{(-)}^{a}{ }_{b}=\hat{\omega}^{a}{ }_{b}-\frac{1}{2} \hat{H}_{\mu}{ }^{a} b d x^{\mu}$, is

$$
\hat{R}_{(-)}^{a b}=d \hat{\omega}_{(-)}^{a b}-\hat{\omega}_{(-)}^{a}{ }_{c} \wedge \hat{\omega}_{(-)}^{c} b .
$$

The field strength $H$ of the Kalb-Ramond 2-form $B$ includes a Chern-Simons term

$$
\hat{H}=d \hat{B}+\frac{\alpha^{\prime}}{4} \hat{\Omega}_{(-)}^{\mathrm{L}},
$$

where

$$
\hat{\Omega}_{(-)}^{\mathrm{L}}=d \hat{\omega}_{(-)}{ }^{a} b \wedge \hat{\omega}_{(-)}{ }^{b} a-\frac{2}{3} \hat{\omega}_{(-)}{ }^{a}{ }_{b} \wedge \hat{\omega}_{(-)^{b} c}{ }^{b} \wedge \hat{\omega}_{(-)^{c} a} a .
$$

Then, the corresponding Bianchi identity reads

$$
d \hat{H}=\frac{\alpha^{\prime}}{4} \hat{R}_{(-)}^{a} b{ }_{b} \wedge \hat{R}_{(-)}^{b} a .
$$

The equations of motion derived from the action (A.1) are

$$
\begin{aligned}
& \hat{R}_{\mu \nu}-2 \nabla_{\mu} \partial_{\nu} \hat{\phi}+\frac{1}{4} \hat{H}_{\mu \rho \sigma} \hat{H}_{\nu}{ }^{\rho \sigma}-\frac{\alpha^{\prime}}{4} \hat{R}_{(-) \mu \rho b}{ }_{b} \hat{R}_{(-) \nu}{ }^{\rho b}{ }_{a}=\mathcal{O}\left(\alpha^{\prime 2}\right), \\
& (\partial \hat{\phi})^{2}-\frac{1}{2} \nabla^{2} \hat{\phi}-\frac{1}{4 \cdot 3 !} \hat{H}^{2}+\frac{\alpha^{\prime}}{32} \hat{R}_{(-) \mu \nu}{ }_{b} \hat{R}_{(-)}{ }^{\mu \nu b}{ }_{a}=\mathcal{O}\left(\alpha^{\prime 2}\right), \\
& d\left(e^{-2 \hat{\phi}} \star \hat{H}\right)=\mathcal{O}\left(\alpha^{2}\right) .
\end{aligned}
$$

The (zeroth-order) supergravity theory can be recovered from these expressions by setting $\alpha^{\prime}=0$. The action includes a tower of corrections of all powers in $\alpha^{\prime}$ due to the

\footnotetext{
${ }^{12}$ Some examples with non-trivial Yang-Mills fields were given in [31, 32, 41].
} 
recursive definition of the Kalb-Ramond field strength, which breaks the supersymmetry of the supergravity theory. The term of quadratic order in curvature at (A.1) was found imposing supersymmetry of the theory at first order in $\alpha^{\prime}$ after inclusion of the ChernSimons term in the field strength [42]. Further corrections of higher power in the curvature $R_{(-)}$of the torsionful spin connection are required to recover supersymmetry order by order. Additional higher-curvature corrections unrelated to the supersymmetrization of the KalbRamond kinetic term are also present, although those appear first at cubic order in $\alpha^{\prime}$.

\section{B Solving the equations of motion}

We consider the ansatz for the 10-dimensional fields introduced in eq. (2.1),

$$
\begin{aligned}
d \hat{s} & =\frac{c_{\infty}}{c} e^{2\left(\hat{\phi}-\hat{\phi}_{\infty}\right)} d s^{2}-c^{2}\left(d z+V / c_{\infty}\right)^{2}-d y^{i} d y^{i}, \\
\hat{H} & =F \wedge\left(c_{\infty} d z+V\right)+H,
\end{aligned}
$$

and we assume the following form of the building blocks

$$
\begin{aligned}
d s^{2} & =\left(1+\alpha^{\prime} a\right)^{2}\left(1+\frac{Q}{r}\right)^{-2} d t^{2}-\left(1+\alpha^{\prime} b\right)^{2}\left(1+\frac{Q}{r}\right)^{2}\left(d r^{2}+r^{2} d \Omega_{(2)}^{2}\right) \\
F & =\frac{Q}{(r+Q)^{2}}\left(1+\alpha^{\prime} d(r)\right) d t \wedge d r+Q\left(1+\alpha^{\prime} e(r)\right) \sin \theta d \theta \wedge d \varphi \\
V & =-\frac{\epsilon Q}{(r+Q)}\left(1+\alpha^{\prime} f(r)\right) d t-\epsilon Q \cos \theta d \varphi \\
H & =\alpha^{\prime} g(r) \sin \theta d t \wedge d \theta \wedge d \varphi \\
\hat{\phi} & =\hat{\phi}_{\infty}+\alpha^{\prime} \delta \hat{\phi}(r) \\
c & =c_{\infty}\left(1+\alpha^{\prime} \delta c(r)\right)
\end{aligned}
$$

where $Q>0$ and $\epsilon= \pm 1$ is a sign. ${ }^{13}$ For $\epsilon=+1$ and $\epsilon=-1$ we have, respectively, the cases 1 and 2 discussed in sections 2.2 and 2.3. In the limit $\alpha^{\prime} \rightarrow 0$, this ansatz reduces to the zeroth-order solution in eq. (2.7). The $\alpha^{\prime}$ corrections are introduced through the functions $a, b, \delta c, d, e, f, g$ and $\delta \phi$, and one can see that this ansatz is general enough in order to solve the first-order-in- $\alpha^{\prime}$ equations.

On the one hand, keeping only the terms up to linear order in $\alpha^{\prime}$, the Bianchi identity (A.5) reads

$$
\begin{aligned}
d \hat{H}-\frac{\alpha^{\prime}}{4} \hat{R}_{(-)}{ }^{a}{ }_{b} & \wedge \hat{R}_{(-)^{b}{ }^{b}} \\
=\alpha^{\prime} \sin \theta & {\left[d t \wedge d r \wedge d \theta \wedge d \varphi \left(\frac{d Q^{2} \epsilon}{(Q+r)^{2}}\right.\right.} \\
& \left.+\frac{Q^{3}(Q-Q \epsilon+r(5+3 \epsilon))}{(Q+r)^{6}}+\frac{Q^{2} \epsilon e^{\prime}}{Q+r}-\frac{Q^{2} \epsilon\left(e+f-(Q+r) f^{\prime}\right)}{(Q+r)^{2}}-g^{\prime}\right) \\
& \left.+Q c_{\infty} d r \wedge d \theta \wedge d \varphi \wedge d z\left(\frac{Q(Q(-1+\epsilon)+3 r(1+\epsilon))}{(Q+r)^{5}}+e^{\prime}\right)\right] .
\end{aligned}
$$

\footnotetext{
${ }^{13}$ One could add an additional function at the magnetic component of the KK vector $V$ taking the form $-\epsilon Q\left(1+\alpha^{\prime} u(r)\right) \cos \theta d \varphi$, but the equations of motion give $u(r)=0$ at this order.
} 
On the other hand, the equations (A.8), (A.7) and the relevant components of Einstein's equations (A.6) yield

$$
\begin{aligned}
& d\left(e^{-2 \hat{\phi}} \star \hat{H}\right)=-Q \alpha^{\prime} \sin \theta d r \wedge d \theta \wedge d \varphi\left(\frac{g \epsilon}{r^{2}}+\left(a^{\prime}-b^{\prime}-d^{\prime}+\delta c^{\prime}+2 \delta \phi^{\prime}\right)\right), \\
& \mathcal{E}_{\hat{\phi}}=\alpha^{\prime}\left(Q^{2}\left(\frac{Q^{2}-12 r^{2}}{8(Q+r)^{8}}+\frac{(a-b-d+e)}{2(Q+r)^{4}}\right)+\frac{\left(r^{2} \delta \phi^{\prime}\right)^{\prime}}{2(Q+r)^{2}}\right), \\
& \mathcal{E}_{t t}=\frac{\alpha^{\prime}}{(Q+r)^{4}}\left[\frac{e Q^{4}}{(Q+r)^{2}}-\frac{2 a Q^{2} r^{2}}{(Q+r)^{2}}+\frac{d Q^{2}(-Q+r)}{Q+r}+\frac{f Q^{2}\left(Q^{2}+r^{2}\right)}{(Q+r)^{2}}\right. \\
& +\frac{Q^{2} r^{2} \delta \mathrm{c}}{(Q+r)^{2}}-\frac{2 Q^{2} r^{2} \delta \phi}{(Q+r)^{2}}-\frac{g Q^{2} \epsilon}{Q+r} \\
& -\frac{Q^{2} r^{2}\left(12 Q r(-1+\epsilon)+6 r^{2}(-1+\epsilon)+Q^{2}(5+17 \epsilon)\right)}{4(Q+r)^{6}} \\
& +\frac{r^{2}\left(Q^{2}-3 Q r-2 r^{2}\right) a^{\prime}}{Q+r}-Q r^{2} b^{\prime}-\frac{Q^{2}\left(Q^{2}+r^{2}\right) f^{\prime}}{Q+r} \\
& +\frac{r(2 Q+r)\left(Q^{2}-Q r+r^{2}\right) \delta \mathrm{c}^{\prime}}{Q+r}-\frac{2 r^{2}\left(-Q^{2}+Q r+r^{2}\right) \delta \phi^{\prime}}{Q+r} \\
& \left.-r^{4} a^{\prime \prime}+Q^{2} r^{2} f^{\prime \prime}+\left(Q^{2} r^{2}+\frac{r^{4}}{2}\right) \delta c^{\prime \prime}-r^{4} \delta \phi^{\prime \prime}\right], \\
& \mathcal{E}_{r r}=\alpha^{\prime}\left[\frac{2 a Q^{2}}{r^{2}(Q+r)^{2}}-\frac{d Q^{2}}{r^{2}(Q+r)^{2}}-\frac{f Q^{2}}{r^{2}(Q+r)^{2}}-\frac{Q^{2} \delta \mathrm{c}}{r^{2}(Q+r)^{2}}+\frac{2 Q^{2} \delta \phi}{r^{2}(Q+r)^{2}}\right. \\
& +\frac{Q^{4}(1+\epsilon)-2 Q^{2} r^{2}(5+\epsilon)}{4 r^{2}(Q+r)^{6}}+\frac{3 Q a^{\prime}}{r(Q+r)}+\frac{(Q+2 r) b^{\prime}}{Q r+r^{2}} \\
& \left.+\frac{Q^{2} f^{\prime}}{r^{2}(Q+r)}-\frac{\delta \mathrm{c}^{\prime}}{r}+\frac{2 \delta \phi^{\prime}}{r}+a^{\prime \prime}+2 b^{\prime \prime}-\frac{\delta \mathrm{c}^{\prime \prime}}{2}+\delta \phi^{\prime \prime}\right], \\
& \mathcal{E}_{z z}=\frac{\alpha^{\prime} c_{\infty}^{2}}{(Q+r)^{2}}\left[-\frac{d Q^{2}}{(Q+r)^{2}}+\frac{e Q^{2}}{(Q+r)^{2}}+\frac{f Q^{2}}{(Q+r)^{2}}\right. \\
& \left.-\frac{3 Q^{2} r^{2} \epsilon}{(Q+r)^{6}}-\frac{Q^{2} f^{\prime}}{Q+r}+2 r \delta \mathrm{c}^{\prime}+r^{2} \delta \mathrm{c}^{\prime \prime}\right] \\
& \mathcal{E}_{t z}=\frac{\alpha^{\prime} c_{\infty}}{(Q+r)^{3}}\left[\frac{g Q}{2(Q+r)}+\frac{d Q^{3} \epsilon}{(Q+r)^{2}}-\frac{e Q^{3} \epsilon}{(Q+r)^{2}}-\frac{f Q^{3} \epsilon}{(Q+r)^{2}}\right. \\
& +\frac{Q^{2} r^{2}(-3 r(-1+\epsilon)+Q(7+\epsilon))}{2(Q+r)^{6}}-\frac{Q r^{2} \epsilon a^{\prime}}{2(Q+r)}+\frac{Q r^{2} \epsilon b^{\prime}}{2(Q+r)}+\frac{Q^{3} \epsilon f^{\prime}}{Q+r} \\
& \left.-\frac{Q r(4 Q+r) \epsilon \delta \mathrm{c}^{\prime}}{2(Q+r)}-\frac{Q r^{2} \epsilon \delta \phi^{\prime}}{Q+r}-\frac{1}{2} Q r^{2} \epsilon f^{\prime \prime}-Q r^{2} \epsilon \delta \mathrm{c}^{\prime \prime}\right], \\
& \mathcal{E}_{\varphi z}=\frac{\alpha^{\prime} c_{\infty} \cos \theta}{(Q+r)^{2}}\left[\frac{3 Q^{3} r^{2}}{(Q+r)^{6}}+\frac{d Q^{3} \epsilon}{(Q+r)^{2}}-\frac{e Q^{3} \epsilon}{(Q+r)^{2}}\right. \\
& \left.-\frac{f Q^{3} \epsilon}{(Q+r)^{2}}+\frac{Q^{3} \epsilon f^{\prime}}{Q+r}-2 Q r \epsilon \delta \mathrm{c}^{\prime}-Q r^{2} \epsilon \delta \mathrm{c}^{\prime \prime}\right] .
\end{aligned}
$$

Note that here we have written nine equations for eight variables, but of course not all of them are independent, since they are related by Bianchi identities. Nevertheless, it is convenient to use all of the equations above in order to simplify the problem. By 
combining the equations in an appropriate way it is possible to find the general solution, which contains a large number of integration constants. These constants are then fixed by the conditions specified in the main text, which, more precisely, can be expressed as follows:

- Regularity at $r=0$ : all of the functions $a, b, \delta c, d, e, f, g, \delta \phi$ are finite at $r=0$.

- Fixed asymptotic values of the scalars: $\delta \hat{\phi}(r) \rightarrow 0, \delta c(r) \rightarrow 0, g(r) \rightarrow 0$ when $r \rightarrow \infty$.

- Asymptotic flatness: $a(r) \rightarrow 0, b(r) \rightarrow 0$ when $r \rightarrow \infty$.

- Absence of additional free charges at order $\alpha^{\prime}: d(r) \rightarrow 0, e(r) \rightarrow 0, f(r) \rightarrow 0$ when $r \rightarrow \infty$.

These conditions completely determine the solution and one finds

$$
\begin{aligned}
a & =\frac{Q^{3}(1-11 \epsilon)+(1-\epsilon)\left(13 Q^{2} r+8 Q r^{2}+2 r^{3}\right)}{80 Q(Q+r)^{4}}, \\
b & =\frac{10 \epsilon Q^{3}+r\left(9 Q^{2}+7 Q r+2 r^{2}\right)(\epsilon-1)}{80 Q(Q+r)^{4}}, \\
\delta c & =\frac{\epsilon Q^{2}}{4(Q+r)^{4}}, \\
d & =\frac{(\epsilon-1)\left(10 Q r+3 r^{2}\right)+Q^{2}(57 \epsilon+63)}{240(Q+r)^{4}}, \\
e & =\frac{Q(Q \epsilon+2 r(1+\epsilon))}{2(Q+r)^{4}}, \\
f & =\frac{r(63 Q+r)(\epsilon-1)}{240(Q+r)^{4}}, \\
g & =0, \\
\delta \hat{\phi} & =\frac{r\left(19 Q^{2}+12 Q r+3 r^{2}\right)(\epsilon-1)+15 Q^{3}(\epsilon+1)}{120 Q(Q+r)^{4}} .
\end{aligned}
$$

For $\epsilon= \pm 1$ one gets the results shown in sections 2.2 and 2.3 .

\section{Wald entropy}

The Wald entropy formula of the ten-dimensional theory is

$$
\mathbb{S}=-2 \pi \int_{\Sigma} d^{8} x \sqrt{|h|} \mathcal{E}^{a b c d} \epsilon_{a b} \epsilon_{c d}
$$

where $\Sigma$ is a cross-section of the horizon with induced metric $h_{\mu \nu}, \epsilon_{a b}$ is the binormal to $\Sigma$ normalized as $\epsilon_{a b} \epsilon^{a b}=-2$ and $\mathcal{E}^{a b c d}$ is defined as

$$
\mathcal{E}^{a b c d}=\frac{g_{s}^{2}}{16 \pi G_{N}^{(10)}} \frac{\delta \mathcal{L}}{\delta \hat{R}_{a b c d}},
$$


where $\mathcal{L}$ is the Lagrangian of the theory (A.1). We can apply this formula to the action of the heterotic theory for the family of regular solutions we study in this article. In order to compute the integrand it is convenient to use flat indices. We define the vielbein

$$
\begin{aligned}
e^{0} & =e^{\phi-\phi_{\infty}} e^{G} d t, & e^{1} & =e^{\phi-\phi_{\infty}} e^{-U} d r, \\
e^{2} & =e^{\phi-\phi_{\infty}} e^{-U} r d \theta, & e^{3} & =e^{\phi-\phi_{\infty}} e^{-U} r \sin \theta d \varphi, \\
e^{4} & =c\left(d z+\frac{V}{c_{\infty}}\right), & e^{i} & =d y^{i} .
\end{aligned}
$$

Here $e^{2 G}=g_{t t}$ and $e^{-2 U}=g_{r r}$, with $g_{\mu \nu}$ the four-dimensional metric in the Einstein frame. The non-vanishing components of the binormal in flat indices are $\epsilon_{01}=-\epsilon_{10}=1$. The volume form entering Wald's formula is

$$
d^{8} x \sqrt{|h|}=d \theta d \varphi d z d^{4} y c_{\infty} e^{2\left(\hat{\phi}-\hat{\phi}_{\infty}\right)} e^{-2 U} r^{2} \sin \theta .
$$

The variation of the Lagrangian with respect to the Riemann tensor contains three non-vanishing contributions. The first one comes from the Einstein-Hilbert term in (A.1), which amounts to

$$
\mathcal{E}_{0}^{a b c d}=\frac{e^{-2\left(\hat{\phi}-\hat{\phi}_{\infty}\right)}}{16 \pi G_{N}^{(10)}} \frac{\delta \hat{R}}{\delta \hat{R}_{a b c d}}=\frac{e^{-2\left(\hat{\phi}-\hat{\phi}_{\infty}\right)}}{16 \pi G_{N}^{(10)}} \eta^{a c} \eta^{b d},
$$

where $\eta^{a b}$ is the inverse flat metric. This term is responsible for the Bekenstein-Hawking leading order term in the entropy, $\mathbb{S}_{0}=A_{\Sigma} / 4 G_{N}^{(10)}$. Following [38], one can see that there are two additional contributions arising from the variation of the Chern-Simons 3-form in the Kalb-Ramond field strength. Each of those is coming from one of the two factors in the decomposition

$$
\hat{\Omega}_{(-)}^{\mathrm{L}}=\mathscr{A}+\hat{\Omega}^{\mathrm{L}},
$$

where $\hat{\Omega}^{\mathrm{L}}$ is the standard Lorentz Chern-Simons term in terms of the spin connection $\hat{\omega}_{b}^{a}$, and

$$
\mathscr{A}=\frac{1}{2} d\left(\hat{\omega}_{b}^{a} \wedge \hat{H}_{a}^{b}\right)+\frac{1}{4} \hat{H}_{b}^{a} \wedge D \hat{H}_{a}^{b}-\hat{R}_{b}^{a} \wedge \hat{H}_{a}^{b}+\frac{1}{12} \hat{H}_{b}^{a} \wedge \hat{H}_{c}^{b} \wedge \hat{H}_{a}^{c} .
$$

Here $\hat{H}_{b}^{a}=\hat{H}_{\mu b}{ }^{a} d x^{\mu}$ and $D \hat{H}^{a}{ }_{b}=d \hat{H}^{a}{ }_{b}+\hat{\omega}^{a}{ }_{c} \wedge \hat{H}^{c}{ }_{b}-\hat{\omega}^{c}{ }_{b} \wedge \hat{H}^{a}{ }_{c}$. Notice that the last term in (A.1) gives no contribution to the entropy, since it is quadratic in the curvature of the torsionful spin connection, which vanishes at the horizon.

Using this rewriting, in first place we get

$$
\mathcal{E}_{1}^{a b c d}=\frac{e^{-2\left(\hat{\phi}-\hat{\phi}_{\infty}\right)}}{16 \pi G_{N}^{(10)}} \frac{\delta}{\delta \hat{R}_{a b c d}}\left(\frac{\alpha^{\prime}}{3 ! 4} \hat{H}^{e f g} \mathscr{A}_{e f g}\right)=\frac{e^{-2\left(\hat{\phi}-\hat{\phi}_{\infty}\right)}}{16 \pi G_{N}^{(10)}} \frac{\alpha^{\prime}}{8} \hat{H}^{a b f} \hat{H}_{f}{ }^{c d} .
$$

To obtain the last correction to the entropy, we notice that when $\mathcal{E}^{a b c d}$ gets contracted with the binormal, the only relevant values of the flat indices $a, \ldots, d$ are 0,1 . Therefore, 
the remaining non-vanishing contribution to the entropy comes from the variation of $\hat{\Omega}_{014}^{L}$, and amounts to

$$
\mathcal{E}_{2}^{a b c d}=\frac{e^{-2\left(\hat{\phi}-\hat{\phi}_{\infty}\right)}}{16 \pi G_{N}^{(10)}} \frac{\delta}{\delta \hat{R}_{a b c d}}\left(\frac{\alpha^{\prime}}{3 ! 4} \hat{H}^{e f g} \Omega_{e f g}^{L}\right)=\frac{e^{-2\left(\hat{\phi}-\hat{\phi}_{\infty}\right)}}{16 \pi G_{N}^{(10)}} \frac{\alpha^{\prime}}{8} \hat{H}^{a b 4} \frac{c}{c_{\infty}} G^{c d},
$$

Putting everything together, Wald's entropy is

$$
\mathbb{S}=\frac{1}{4 G_{N}^{(10)}} \int d \theta d \varphi d z d^{4} y c_{\infty} e^{-2 U} r^{2} \sin \theta\left[1-\frac{\alpha^{\prime}}{4} \hat{H}^{014}\left(\hat{H}_{4}{ }^{01}+\frac{c}{c_{\infty}} G^{01}\right)\right] .
$$

\section{Case 3: $q_{A} \cdot q_{V}=0$}

As we have seen in the main text, the cases $q_{A} \cdot q_{V}>0$ and $q_{A} \cdot q_{V}<0$ are qualitatively very different when higher-curvature corrections are incorporated. It is interesting to consider the transition between one and another, $q_{A} \cdot q_{V}=0$. In particular let us consider the configuration $q_{V}=p_{V}=0, q_{A}=p_{A}=Q / \sqrt{2}$. Then we find the following solution,

$$
\begin{aligned}
d s^{2} & =A^{2}\left(1+\frac{Q}{\rho}\right)^{-2} d t^{2}-B^{2}\left(1+\frac{Q}{\rho}\right)^{2}\left(d \rho^{2}+\rho^{2} d \Omega_{(2)}^{2}\right), \\
F & =\frac{c}{c_{\infty}} \frac{e^{2\left(\hat{\phi}-\hat{\phi}_{\infty}\right)} \sqrt{2} Q A}{(\rho+Q)^{2} B} d t \wedge d \rho+\sqrt{2} Q E \sin \theta d \theta \wedge d \varphi \\
V & =\frac{\alpha^{\prime} \rho^{2}\left(Q\left(11 Q^{2}+15 Q \rho+6 \rho^{2}\right)-6(Q+\rho)^{3} \log \left(1+\frac{Q}{\rho}\right)\right)}{3 \sqrt{2} Q^{2}(Q+\rho)^{5}} d t, \\
\hat{\phi} & =\hat{\phi}_{\infty}+\frac{\alpha^{\prime}}{1120 Q^{2}(Q+\rho)^{4}}\left[Q\left(-189 Q^{3}-196 Q^{2} \rho+1646 Q \rho^{2}+1304 \rho^{3}\right)\right. \\
c(\rho) & =c_{\infty}+\frac{\alpha^{\prime}}{560 Q^{2}(Q+\rho)^{4}}\left[Q\left(259 Q^{3}+896 Q^{2} \rho+1614 Q \rho^{2}+876 \rho^{3}\right)\right. \\
H & =-\frac{\alpha^{\prime} Q^{2} \rho^{2}}{(Q+\rho)^{5}} d t \wedge \sin \theta d \theta \wedge d \varphi-F \wedge V,
\end{aligned}
$$

where

$$
\begin{aligned}
& A=1+\alpha^{\prime} \frac{83 Q^{3}+189 Q^{2} \rho+84 Q \rho^{2}+6 \rho^{3}-60(Q+\rho)^{3} \log \left(1+\frac{Q}{\rho}\right)}{480 Q(Q+\rho)^{4}} \\
& B=1+\alpha^{\prime} \frac{Q(5 Q+3 \rho)\left(6 Q^{2}+13 Q \rho+18 \rho^{2}\right)-60 \rho(Q+\rho)^{3} \log \left(1+\frac{Q}{\rho}\right)}{480 Q^{2}(Q+\rho)^{4}} \\
& E=1+\frac{\alpha^{\prime} Q(Q+4 \rho)}{4(Q+\rho)^{4}}
\end{aligned}
$$


We observe that there are logarithmic singularities that cannot be avoided. Now, the charges of the solution are indeed $q_{A}=p_{A}=Q / \sqrt{2}$. Thus, $Q$ still represents the total charge $Q=\sqrt{q_{A}^{2}+p_{A}^{2}}$. In addition, we note that the KK vector field $V$ does not carry any charge. However, we get

$$
g_{\rho \rho}=1+\frac{1}{\rho}\left(2 Q-\frac{\alpha^{\prime}}{40 Q}\right)+\ldots
$$

Therefore, the mass is

$$
M=Q-\frac{\alpha^{\prime}}{80 Q}+\ldots \Rightarrow \frac{Q}{M}=1+\frac{\alpha^{\prime}}{80 M^{2}}+\mathcal{O}\left(\alpha^{\prime 2}\right) .
$$

Open Access. This article is distributed under the terms of the Creative Commons Attribution License (CC-BY 4.0), which permits any use, distribution and reproduction in any medium, provided the original author(s) and source are credited.

\section{References}

[1] R. Kallosh et al., Supersymmetry as a cosmic censor, Phys. Rev. D 46 (1992) 5278 [hep-th/9205027] [INSPIRE].

[2] S. Ferrara, R. Kallosh and A. Strominger, $N=2$ extremal black holes, Phys. Rev. D 52 (1995) R5412 [hep-th/9508072] [INSPIRE].

[3] A. Strominger and C. Vafa, Microscopic origin of the Bekenstein-Hawking entropy, Phys. Lett. B 379 (1996) 99 [hep-th/9601029] [INSPIRE].

[4] C.G. Callan and J.M. Maldacena, D-brane approach to black hole quantum mechanics, Nucl. Phys. B 472 (1996) 591 [hep-th/9602043] [INSPIRE].

[5] S. Ferrara and R. Kallosh, Supersymmetry and attractors, Phys. Rev. D 54 (1996) 1514 [hep-th/9602136] [INSPIRE].

[6] S. Ferrara and R. Kallosh, Universality of supersymmetric attractors, Phys. Rev. D $\mathbf{5 4}$ (1996) 1525 [hep-th/9603090] [INSPIRE].

[7] A. Sen, Black hole entropy function and the attractor mechanism in higher derivative gravity, JHEP 09 (2005) 038 [hep-th/0506177] [INSPIRE].

[8] S.D. Mathur, The Fuzzball proposal for black holes: an elementary review, Fortsch. Phys. 53 (2005) 793 [hep-th/0502050] [INSPIRE].

[9] K. Papadodimas and S. Raju, State-dependent bulk-boundary maps and black hole complementarity, Phys. Rev. D 89 (2014) 086010 [arXiv:1310.6335] [INSPIRE].

[10] T. Banks and N. Seiberg, Symmetries and strings in field theory and gravity, Phys. Rev. D 83 (2011) 084019 [arXiv:1011.5120] [INSPIRE].

[11] L. Susskind, Trouble for remnants, hep-th/9501106 [INSPIRE].

[12] N. Arkani-Hamed, L. Motl, A. Nicolis and C. Vafa, The string landscape, black holes and gravity as the weakest force, JHEP 06 (2007) 060 [hep-th/0601001] [INSPIRE].

[13] C. Vafa, The string landscape and the swampland, hep-th/0509212 [INSPIRE].

[14] E. Palti, The swampland: introduction and review, Fortsch. Phys. 67 (2019) 1900037 [arXiv: 1903.06239] [INSPIRE]. 
[15] L. Aalsma, A. Cole and G. Shiu, Weak gravity conjecture, black hole entropy and modular invariance, arXiv:1905.06956 [INSPIRE].

[16] Y. Kats, L. Motl and M. Padi, Higher-order corrections to mass-charge relation of extremal black holes, JHEP 12 (2007) 068 [hep-th/0606100] [INSPIRE].

[17] G.J. Loges, T. Noumi and G. Shiu, Thermodynamics of $4 D$ dilatonic black holes and the weak gravity conjecture, arXiv:1909.01352 [INSPIRE].

[18] C. Cheung and G.N. Remmen, Infrared consistency and the weak gravity conjecture, JHEP 12 (2014) 087 [arXiv: 1407.7865] [INSPIRE].

[19] Y. Hamada, T. Noumi and G. Shiu, Weak gravity conjecture from unitarity and causality, Phys. Rev. Lett. 123 (2019) 051601 [arXiv:1810.03637] [INSPIRE].

[20] B. Bellazzini, M. Lewandowski and J. Serra, Amplitudes' positivity, weak gravity conjecture and modified gravity, Phys. Rev. Lett. 123 (2019) 251103 [arXiv:1902.03250] [INSPIRE].

[21] A.M. Charles, The weak gravity conjecture, $R G$ flows and supersymmetry, arXiv: 1906.07734 [INSPIRE].

[22] C. Cheung, J. Liu and G.N. Remmen, Proof of the weak gravity conjecture from black hole entropy, JHEP 10 (2018) 004 [arXiv:1801.08546] [INSPIRE].

[23] C. Cheung, J. Liu and G.N. Remmen, Entropy bounds on effective field theory from rotating dyonic black holes, Phys. Rev. D 100 (2019) 046003 [arXiv:1903.09156] [INSPIRE].

[24] G. Goon and R. Penco, A universal relation between corrections to entropy and extremality, arXiv: 1909.05254 [INSPIRE].

[25] G.W. Gibbons, Antigravitating black hole solitons with scalar hair in $N=4$ supergravity, Nucl. Phys. B 207 (1982) 337 [inSPIRE].

[26] G.W. Gibbons and K.-i. Maeda, Black holes and membranes in higher dimensional theories with dilaton fields, Nucl. Phys. B 298 (1988) 741 [INSPIRE].

[27] D. Garfinkle, G.T. Horowitz and A. Strominger, Charged black holes in string theory, Phys. Rev. D 43 (1991) 3140 [Erratum ibid. D 45 (1992) 3888] [INSPIRE].

[28] A. Strominger, Macroscopic entropy of $N=2$ extremal black holes, Phys. Lett. B 383 (1996) 39 [hep-th/9602111] [INSPIRE].

[29] S. Ferrara, G.W. Gibbons and R. Kallosh, Black holes and critical points in moduli space, Nucl. Phys. B 500 (1997) 75 [hep-th/9702103] [INSPIRE].

[30] S. Ferrara and R. Kallosh, On $N=8$ attractors, Phys. Rev. D 73 (2006) 125005 [hep-th/0603247] [INSPIRE].

[31] P.A. Cano, P. Meessen, T. Ortín and P.F. Ramírez, $\alpha^{\prime}$-corrected black holes in string theory, JHEP 05 (2018) 110 [arXiv: 1803.01919] [INSPIRE].

[32] P.A. Cano et al., Beyond the near-horizon limit: stringy corrections to heterotic black holes, JHEP 02 (2019) 192 [arXiv: 1808.03651] [INSPIRE].

[33] A.M. Charles, F. Larsen and D.R. Mayerson, Non-renormalization for non-supersymmetric black holes, JHEP 08 (2017) 048 [arXiv: 1702.08458] [INSPIRE].

[34] B.A. Campbell, N. Kaloper and K.A. Olive, Classical hair for Kerr-Newman black holes in string gravity, Phys. Lett. B 285 (1992) 199 [INSPIRE]. 
[35] P.A. Cano et al., $\alpha^{\prime}$ corrections of Reissner-Nordström black holes, JHEP 02 (2020) 031 [arXiv: 1910.14324] [INSPIRE].

[36] R.R. Khuri and T. Ortín, A nonsupersymmetric dyonic extreme Reissner-Nordstrom black hole, Phys. Lett. B 373 (1996) 56 [hep-th/9512178] [INSPIRE].

[37] P.A. Cano, P.F. Ramírez and A. Ruipérez, The small black hole illusion, arXiv:1808.10449 [INSPIRE].

[38] F. Faedo and P.F. Ramirez, Exact charges from heterotic black holes, JHEP 10 (2019) 033 [arXiv: 1906.12287] [INSPIRE].

[39] D. Astefanesei, R. Ballesteros, D. Choque and R. Rojas, Scalar charges and the first law of black hole thermodynamics, Phys. Lett. B 782 (2018) 47 [arXiv:1803.11317] [INSPIRE].

[40] E.A. Bergshoeff and M. de Roo, The quartic effective action of the heterotic string and supersymmetry, Nucl. Phys. B 328 (1989) 439 [inSPIRE].

[41] S. Chimento et al., On a family of $\alpha^{\prime}$-corrected solutions of the Heterotic Superstring effective action, JHEP 07 (2018) 080 [arXiv: 1803.04463] [INSPIRE].

[42] E. Bergshoeff and M. de Roo, Supersymmetric Chern-Simons terms in ten-dimensions, Phys. Lett. B 218 (1989) 210 [INSPIRE]. 